\title{
Fronteras de EFICIENCIA PARA OPERAdores DE DECISIONES
}

(1) Eduardo Raffo Lecca

(2) Edgar Ruiz Lizama

\section{RESUMEN \\ El artículo destaca la metodología del análisis envolvente de datos DEA (Del ingles DATA Envelopment Analysis) como una herramienta del "Benchmarking" para cuantificar o medir la eficiencia de las organizaciones. Se exponen desde conceptos generales, hasta las funciones de frontera de eficiencia mostrando su sustento matemático y también los alcances y bondades del DEA.}

Palabras Clave: Análisis envolvente de datos, eficiencia, frontera de eficiencia, análisis de regresión.

\section{EFFICIENCY FRONTIERS FOR DECISION OPERATORS \\ ABSTRACT}

This work highlights the Data Envelopment Analysis (DEA) Methodology as a benchmarking tool suitable to quantify or measure the efficiency of an organization. From basic concepts to efficiency frontier functions are explained here, showing the mathematical fundamentals, together with the DEA goodness and scope.

Key words: Data envelopment analysis, efficiency, efficiency frontier, regression analysis.

(1) Ingeniero Industrial. Profesor del Departamento de Ingeniería de Sistemas e Informática, UNMSM. Ingenieria de Sistemas e Informe

(2) Ingeniero Industrial. Profesor del Departamento de Ingeniería de Sistemas e Informática, UNMSM. E-mail: eruizl@unmsm.edu.pe

\section{INTRODUCCIÓN}

La organización debe medir; porque si no mide, no administra, por lo tanto no hay mejora, continua el desorden, los conflictos y la depresión. La organización obtendrá lo que mida y reconozca. Todo lo que hace una organización es importante dentro y fuera por ello debe medirse.

En el ámbito de la economía, la eficiencia hace referencia a un juicio acerca de la relación entre los medios empleados y los fines obtenidos. La eficiencia orientada a producción, responde a la pregunta acerca de cuánto podemos expandir la producción sin alterar la cantidad de insumos necesarios, empleando una función de producción que permita alcanzar una medida de eficiencia técnica.

El interés por el análisis de las fronteras de eficiencia económica, ha crecido rápidamente durante los últimos tiempos. Existiendo numerosas metodologías y aplicaciones con referencia a la medición de la efectividad. Desde las ideas de Farrell; Charnes, Cooper y Rhodes en 1978; desarrollaron el Análisis Envolvente de Datos DEA. En el cual se busca establecer que organizaciones de la muestra determinan la superficie envolvente o frontera de producción eficiente.

\section{EFICIENCIA Y PERFORMANCE}

Desde los tiempos inmemorables, las personas han estado contando y registrando resultados. Una inclinación natural del hombre es conocer el "How many" y el "How much". Una vez que las respuestas han sido registradas, el siguiente paso es comparar el conjunto de números con otros. Esta habilidad de comparar forma parte de nuestra vida diaria.

Cinco, son los principios fundamentales de la administración que toda organización, independientemente del tamaño, la actividad y los recursos que posea; debe realizar en la práctica diaria, si el objetivo es crecer ser competitivo y rentable: (a) medir, (b) aprender, (c) innovar, (d) poner en marcha lo aprendido y (e) mejoramiento continuo.

\section{Conceptos generales}

Preguntas como: ¿Cuál es el más grande de los almacenes?, ¿cuál es el mejor producto?, ¿qué hospital ofrece el mejor servicio?; forman parte de los esfuerzos en los negocios y gobiernos, para sus procesos de contar, medir y comparar.

Estadísticas gubernamentales como: índice de la pobreza, población económicamente activa, balanza de pagos; son necesarias para comparar la 
efectividad relativa de un periodo a otro. Una vez obtenidas las estadísticas globales y empezar a comparar el resultado, para pequeños grupos, nos encontramos con el problema de la determinación de una base equitativa para las comparaciones.

En realidad el problema, de contar para una variedad de características, a menudo representa un problema de conflictos de objetivos.

Desde otra óptica, cuando un miembro de un grupo, se pregunta “¿Cómo los otros están haciendo?” y “¿Cuánto mas pueden mejorar?”, nos encontramos con el tema de la performance.

\section{Ratios en contabilidad y finanzas}

Los ratios del balance general, son los referidos a la evolución a corto plazo del balance de la empresa. Estos ratios son principalmente:

- Apalancamiento

- Fondo de maniobra sobre ventas

- Fondo de maniobra sobre activo circulante

- Rotaciones

- Prueba ácida

Los ratios en estados de resultados intentan obtener una medida de la rentabilidad de la empresa. Sobre todo se usan en las cuentas de resultados estimadas para el futuro, tomando como base de comparación las del pasado. Los más importantes son:

- Flujo de caja sobre deuda

- Tasa de amortización contable

- Beneficio neto sobre recursos propios

- Rentabilidad Económica, etc.

Cada uno de estos ratios, dan una pintura parcial e incompleta de la salud de una compañía.

En atención a esta temática, los economistas han desarrollado un indicador viable que es conocido como z-score. Este es una composición de sumas ponderadas de ratios. El modelo de Altman, es un modelo teórico, utilizado para predecir quiebras.

Cuadro 1. Producción planeada vs producción alcanzada

\begin{tabular}{|c|cccc|}
\hline \multirow{2}{*}{ Compañía } & \multicolumn{2}{|c}{ Presupuesto } & \multicolumn{2}{c|}{ Real } \\
\cline { 2 - 5 } & costo & producción & costo & producción \\
\hline A & 100 & 360 & 120 & 400 \\
B & 100 & 360 & 80 & 320 \\
C & 100 & 360 & 100 & 360 \\
\hline
\end{tabular}

Elaboración propia, 2005.

\section{EFICIENCIA, EFECTIVIDAD Y ECONOMÍA}

Existe cierta confusión en los términos eficiencia, efectividad y performance. Brevemente diremos:

- Efectividad: relativo a las metas alcanzadas.

- Economía: relativo a la entrada predeterminada.

- Eficiencia: relativo al uso de un recurso en alcanzar una salida.

\section{Combinando en los términos una ecuación:}

Eficiencia alcanzada $=$ Efectividad $\times$ Economía $\times$ Eficiencia planeada

Donde las entradas cubren los recursos y las salidas la alcanzada. La eficiencia alcanzada es a veces denominada "valor del dinero". En el Cuadro1, se presentan los datos de producción planeada y producción alcanzada.

En este ejemplo, se puede apreciar que una simple entrada o simple salida, permite una fácil y equívoca administración y la presentación de diferentes aseguramientos, dependiendo de diferentes puntos de vistas. Cualquier aseguramiento de la eficiencia es subjetivo. Esto quiere decir, que es mucho mas fácil, efectuar mediciones económicas y efectividad, al menos de factor por factor.

En el ámbito de la economía, la eficiencia hace referencia a un juicio acerca de la relación entre los medios empleados y los fines obtenidos. Una técnica, un procedimiento una persona o un sistema de producción será eficiente si, una determinada disponibilidad de entrada, es capaz de producir la máxima cantidad de salida (output) posible o alternativamente, si para alcanzar determinado nivel de salida se utiliza la menor cantidad de entrada (input).

La unidad organizativa denominada DMU (Decisión Making Unit), se utiliza para medir la eficiencia, puede ser de variados tipos: unidades productivas dentro de una organización, firmas o empresas dentro de un sector, personas dentro de una organización.

Cuadro 2. Eficiencia planeada vs. Eficiencia alcanzada

\begin{tabular}{|c|c|c|c|c|}
\hline Compañía & \begin{tabular}{|l} 
Eficiencia \\
Planeada \\
\end{tabular} & Economía & Efectividad & $\begin{array}{l}\text { Eficiencia } \\
\text { alcanzada }\end{array}$ \\
\hline$A$ & 3.6 & 0.83 & 1.11 & 3.33 \\
\hline B & 3.6 & 1.25 & 0.89 & 4.00 \\
\hline C & 3.6 & 1.00 & 1.00 & 3.60 \\
\hline
\end{tabular}

Elaboración propia, 2005. 


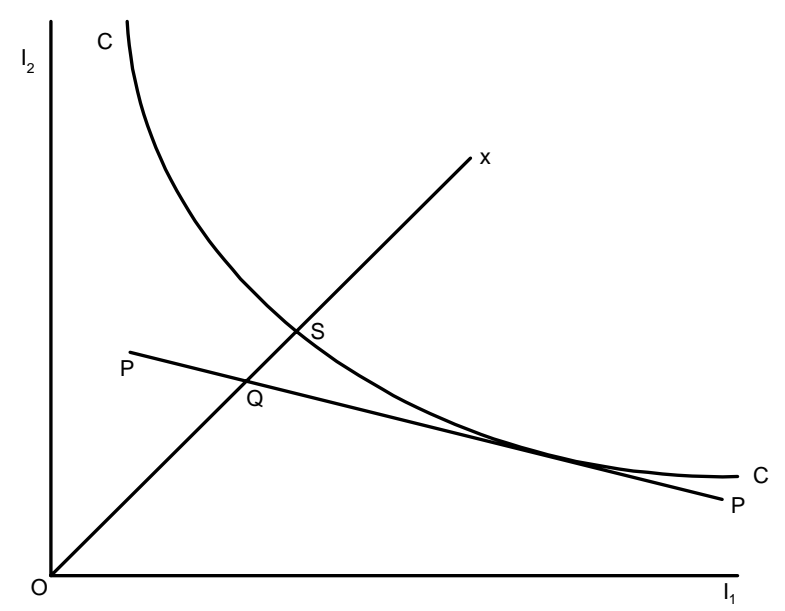

Figura1. Curva CC para los factores $I_{1}, I_{2}$

Funciones de fronteras de eficiencia Los economistas usan el término "eficiencia productiva", para describir como una unidad organizativa, desarrolla la utilización de recursos para generar la utilización de recursos.

El análisis de eficiencia productiva comenzó en los años 50 con los trabajos de Koopmans. A él se debe la primera definición de la eficiencia productiva: una unidad que utiliza varios insumos para producir varios productos es técnicamente eficiente si, y solamente si, es imposible que insuma más de cualquier insumo sin producir menos de algún otro producto o usar mas de algún otro insumo.

Debreu y Shepard, introdujeron la noción de una función de distancias como una manera de modelar las tecnologías con múltiples salidas.

La primera aproximación cuantitativa al concepto de eficiencia se debe a Farrell, un pionero en este campo inspirado en los trabajos de Koopmans; quien demostró que la eficiencia global, puede ser descompuesta en eficiencia técnica y eficiencia asignativa. Se habla de eficiencia técnica o productiva, cuando a partir de una determinada tecnología de producción fija, se consigue alcanzar el máximo nivel de salida posible. Por otro lado la eficiencia asignativa, se define para aquella situación en que, conocidos los precios relativos de las diferentes entradas utilizados en el proceso productivo y partiendo del mejor supuesto de que la tecnología de producción puede cambiar, si emplea la mejor combinación de entradas que permite alcanzar un determinado nivel de salida, con el menor costo. En definitiva, la eficiencia global viene referida al producto de la eficiencia técnica y de la asignativa.

En la Figura 1, se observa que la salida es producto de los factores $I_{1}, I_{2}$, con la curva CC empezando una

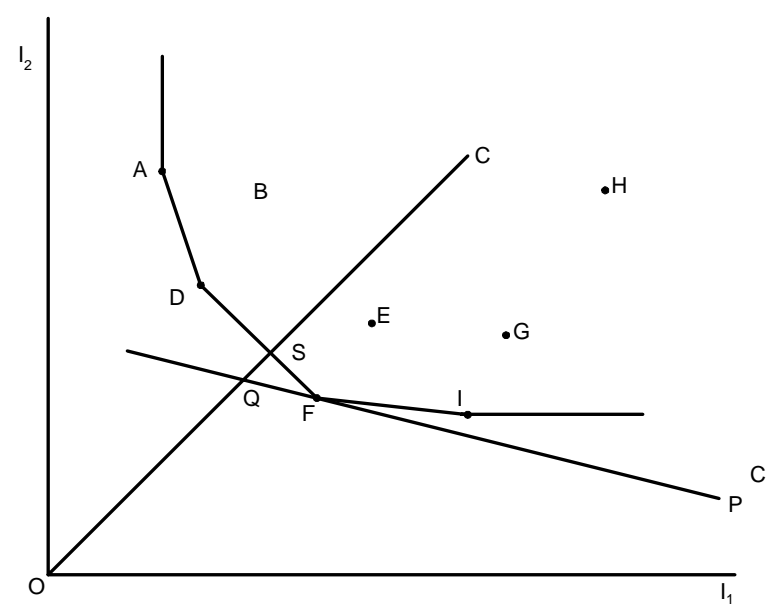

Figura 2. Función de producción con frontera de eficienciaADFI

salida isocuanta. La línea PP representa el plano del costo de minimización y la eficiencia global de unidades X es medida por OQ/OX. La eficiencia técnica $(T)$, medida como la distancia radial de $X$ que está al frente de la isocuanta y la eficiencia asignativa (A), medida como la distancia radial desde el plano de la minimización del costo; es decir:

$$
T=\frac{O S}{O X} \quad \text { y } \quad A=\frac{O Q}{O S}
$$

Se tiene entonces que la eficiencia global, puede ser computada desde A y T, como sigue:

$$
E=\frac{O Q}{O X}=\frac{O Q}{O S} \times \frac{O S}{O X}=A \times T
$$

A partir del trabajo de Farrell, otros autores han propuesto conceptos alternativos. Los trabajos de Forsund y Hjalmarsson, descomponen la eficiencia en técnica, asignativa y de escala. La eficiencia de escala es relevante cuando la tecnología de producción presenta rendimientos de escala variables.

En cuanto a su medición, cabe señalar que la eficiencia es un concepto relativo, de forma que el resultado de una unidad económica debe ser comparado con un estándar. En este sentido, la medición de la eficiencia requiere de dos etapas. En primer lugar la determinación de una función de referencia estándar que indique, dada una tecnología de producción fija, el máximo nivel de salida alcanzable a partir de diferentes combinaciones de entradas.

La función de referencia o función frontera, puede ser tanto una función de producción como una función de costos, o incluso de beneficios. La segunda etapa consiste en comparar los resultados obtenidos por cada unidad de producción con la frontera estándar, de forma que las desviaciones existentes quedarán caracterizadas como comportamientos ineficientes. 
La eficiencia orientada a producción, responde a la pregunta acerca de cuánto podemos expandir la producción sin alterar la cantidad de insumos necesarios, empleando una función de producción que permite alcanzar una medida de eficiencia técnica.

\section{Estimación de las funciones de frontera}

Para la estimación de las funciones de frontera, se han propuesto dos tipos básicos de modelos: paramétricos (cualquiera determinista o estocástica) y no paramétricos. La diferencia entre ambos es que los primeros especifican una relación funcional entre las entradas y las salidas, en tanto que los segundos no imponen ninguna restricción.

Los indicadores de eficiencia, se miden a través de las desviaciones respecto de la frontera de producción que permite aproximar en el nivel empírico la función de producción.

Una frontera es determinista, porque atribuyen toda la aleatoriedad al término de la ineficacia técnica, motivo por el cual ignoran la naturaleza estocástica de la producción, así como la posible existencia de perturbaciones de tipo exógenas.

Las fronteras estocásticas, se caracterizan por la naturaleza estocástica de la producción; y es especificada de la siguiente manera:

$$
y=f(x)+\varepsilon, \quad \varepsilon=v-u
$$

Donde $v$ es la perturbación aleatoria, distribuida con media cero.

Independiente de la naturaleza, se pueden plantear función paramétrica de los factores productivos; haciendo uso de la programación matemática o las técnicas econométricas: mínimos cuadrados y máxima verosimilitud. El uso de la programación matemática en la estimación de las fronteras de producción, se inicia usando la técnica del tipo Cobb-Douglas, desde el modelo

$$
\operatorname{Lny}=B_{0}+B_{j} \operatorname{Lnx} x_{j}-u
$$

Donde $x_{i}$, es el insumo j-ésimo, en la función de producción.

La eficiencia productiva, se alcanza cuando se incrementa la producción con los insumos disponibles. La ventaja del propósito paramétrico, es que cualquier hipótesis puede ser probada con el rigor de la estadística.

En muchos casos, no se conoce la forma de la función de producción; y no es apropiado referirse en términos de función de producción. Esto es claro en las organizaciones gubernamentales, tales como salud y educación; como también en las organizaciones del sector privado.

En el análisis no paramétrico, no existen asunciones sobre la función de producción. Una función de mejor práctica, es construida a partir de las observaciones entre las entradas y salidas. Esta será necesariamente una función lineal por piezas. En la Figura 2, se presenta una función de producción; donde la frontera de eficiencia corresponde a las líneas: A-D-F-I. Esta es la base para el desarrollo de fronteras, para la estimación de la eficiencia técnica.

\section{ANÁLISIS ENVOLVENTE DE DATOS}

En los últimos años, el Análisis Envolvente de Datos se ha convertido en el método de Benchmarking muy utilizado por las empresas. La principal ventaja de DEA consiste en que no está basado en el conocimiento de la función de producción. Corresponde a un método no paramétrico, permitiendo así modelos más ricos y no dependientes del conocimiento de los precios de los factores de producción.

DEA encuentra el conjunto de empresas eficientes a partir de las cuales, mediante combinaciones lineales, obtiene la envolvente o frontera. Esto representa una ventaja por su mayor flexibilidad, aunque para muchos su inconveniente fundamental radica en la falta de propiedades estadísticas de los resultados obtenidos con la programación lineal. Sin embargo, a su favor está la factibilidad de incorporar las economías de escala en el análisis; ventaja importante que justifica su elección.

Metodología de fronteras en Benchmarking

El Benchmarking es un método empleado dentro de las organizaciones para monitorear su funcionamiento y evaluar sus potenciales mejoras de eficiencia.

En los procesos de regulación con incentivo, la eficiencia se mide en relación a un previo Benchmarking y su resultado proporciona la información necesaria para comparar la operación de los negocios y permite identificar las acciones requeridas para impulsar mejoras en su eficiencia.

En el desarrollo de diferentes metodologías, una fuente primaria de diferenciación ha sido el grado de énfasis puesto en la relación entre los ingresos y los costos. Esta diferenciación permite medir con respecto a las referencias que se encuentran ligadas o desligadas a los costos de las empresas. 
Cuando se compara el funcionamiento de las empresas, es común hacerlo en consideración a que si son más o menos eficientes, noción que se encuentra estrechamente conectada a los factores que determinan la función de producción y que es determinada con los índices o medidas de eficiencia de productividad.

El cálculo de la utilidad, para un empresa que utiliza una sola entrada para producir una sola salida, es sumamente fácil, en cambio cuando, en los casos de empresas que utilizan varias entradas para producir varias salidas, es necesario agrupar, como una suma ponderada, todas las salidas en el numerador y todas las entradas en el denominador, de tal manera de continuar obteniendo la productividad como la razón entre dos cantidades que están adecuadamente escaladas.

La eficiencia productiva, esta definida como la capacidad de la empresa para producir un producto a costo mínimo. Para alcanzar el costo mínimo, la empresa debe emplear sus entradas en forma eficiente (eficiencia técnica) y debe elegir la combinación de los insumos correctamente, dado el precio relativo de éstos (eficiencia de asignación).

La obtención de medidas de eficiencia para un grupo de organizaciones, mediante el análisis de datos orientados a determinar una máxima producción o mínimo costo (fronteras), implica una gran variedad de métodos entre los cuales se cuentan las técnicas econométricas y de programación matemática.

\section{Análisis de eficiencia productiva}

Para Farrell, la eficiencia técnica significa las desviaciones observadas respecto a una frontera idealizada o isocuanta. El enfoque de Farrell es un tratamiento econométrico, en la cual la eficiencia se expresa por los residuales de un modelo de regresión. Farrell estimó la frontera paramétrica empleando modelos de programación lineal.

Desde las ideas de Farrell; Charnes, Cooper y Rhodes en 1978, desarrollan el Análisis de Datos Envolventes o DEA. En la cual se busca establecer cuáles organizaciones de la muestra determinan la superficie envolvente o frontera de producción eficiente. La distancia radial de una organización hacia la frontera provee la medida de eficiencia. Por tanto, aquellas empresas que están sobre la frontera (las que la determinan) son consideradas eficientes, en cambio aquellas que se encuentran alejadas de la frontera son consideradas ineficientes. En esta metodología, la búsqueda de la frontera se realiza con técnicas de programación lineal.
Definidas las metodologías para estimar la frontera de eficiencia, queda por especificar el tipo de frontera a estimar, lo que puede hacerse a partir de una función de producción o a partir de una función de costo. Una función de producción relaciona las cantidades productivas en función de los insumos utilizados, en cambio, una función de costo relaciona el costo total de producción en función de los niveles de productos y el precio de los insumos.

El interés por el análisis de las fronteras de eficiencia económica, ha crecido rápidamente durante los últimos tiempos. Existiendo numerosas metodologías y aplicaciones con referencia a la medición de la efectividad.

En general, son dos las metodologías desarrolladas para medir la eficiencia: la econométrica y la de programación matemática. En ambos casos la eficiencia de una determinada empresa es el resultado de una medida relativa de distancia respecto a una frontera que representa el límite práctico de la eficiencia.

Las empresas que constituyen la mejor práctica componen lo que se denomina frontera eficiente o isocuanta, término que alude el hecho de que no es posible ser más eficiente que las empresas situadas en dicha frontera.

\section{CONCLUSIONES}

Estadísticas gubernamentales como: índice de la pobreza, población económicamente activa, balanza de pagos; son necesarias para comparar la relativa efectividad de un periodo a otro.

En realidad el problema, de contar para una variedad de características, a menudo representa un problema de conflictos de objetivos.

Los ratios, dan una pintura parcial e incompleta de la salud de una compañía.

Los economistas usan el término "eficiencia productiva", para describir como una unidad organizativa, desarrolla la utilización de recursos para generar la utilización de recursos.

Para la estimación de las funciones de frontera, se han propuesto dos tipos básicos de modelos: (a) paramétricos (cualquiera determinista o estocástica) y (b) no paramétricos.

El Análisis de Datos Envolventes o DEA, busca establecer cuáles organizaciones de la muestra determi- 
nan la superficie envolvente o frontera de producción eficiente. La distancia radial de una organización hacia la frontera provee una medida de la eficiencia.

\section{BIBLIOGRAF IA}

1. Banker, R.D.; C.A.K. y Schmidt, P. (1984). Some Models for Estimating Technical and Scale Inefficiencies in Data Envelopment Analysis. Management Science, Vol. 30, No 9, pp. 1078-1092.

2. Charnes, A.; Cooper, W. W. y Rhodes, E. (1981). Evaluating Program and Managerial Efficiency: An Application of Data Envelopment Analysis to program Follow Through. Management Science, Vol. 27, No 6, pp. 668-697.

3. Charnes, A.; Cooper, W. W. y Rhodes, E. (1978). Measuring the Efficiency of Decision Making Units. European Journal of Operational Research 2: pp. 429-444.
4. Farrell, M.J. (1957). The Measurement of Productive Efficiency. Journal of the Royal Statistical Society, Serie A (general), Vol. 120 (III), pp. 253-281.

5. Farrell, M.J. y Fieldhouse, M. (1962). Estimating efficient production functions under increasing return to scale. Journal of the Royal Statistical Society, Serie A, Vol. 125.

6. Forsund, F.R. y Hjalmarsson, L. (1974). On the Measurement of Productive Efficiency. The Swedish Royal of Economics, Vol. 76, No 2, pp. 141-154.

7. Forsund, F.R., C.A.K. P. Schmidt, (1980). A Survey of Frontier Functions and of their Relations to Efficiency Measurement. Journal of Econometrics, Vol. 13, pp. 5-80.

8. Porter, Michael E. (1998). Ventaja Competitiva. Compañía Editorial Continental, México. 